Erratum

\title{
A novel vehicle-like drug delivery 3D printing scaffold and its applications for a rat femoral bone repairing in vitro and in vivo: Erratum
}

Hui Wang1 ${ }^{1 *}$, Zhengwei Deng $2,5^{*}$, Jing Chen ${ }^{2,5^{*}}$, Xin Qi², Libing Pang 3 , Bocai Lin 1 , Yan Teik Yuin Adib ${ }^{1,4}$, Na Miao $^{6}$, Deping Wang ${ }^{3}$, Yadong Zhang ${ }^{2,5 \bowtie}$, Jiusheng $\mathrm{Li}^{1}{ }^{\bowtie}$, Xiangqiong Zeng ${ }^{\bowtie}$

1. Laboratory for Advance Lubricating Materials, Shanghai Advanced Research Institute, Chinese Academy of Sciences, Shanghai 201210, China.

2. Department of Orthopedics, Fengxian District Central Hospital Affiliated of Shanghai University of Medicine\&Health Sciences, 279 zhouzhu road, Shanghai 220120, People's Republic of China.

3. School of Materials Science and Engineering, Tongji University, Shanghai 201804, China.

4. School of Life Science \& Chemical Technology, Ngee Ann Polytechnic, Singapore 599489

5. Graduate School, Shanghai University of Traditional Chinese Medicine, Shanghai 201203, China

6. Department of Pediatrics, Maternal and Child Health Hospital of Zaozhuang City, Shandong, China.

*The first authors contributed equally to this work

$\bowtie$ Corresponding authors: Jiusheng Li: E-mail: lijs@sari.ac.cn; Xiangqiong Zeng: E-mail: zengxq@sari.ac.cn; Yadong Zhang: E-mail: zhangyadong6@126.com

(C) The author(s). This is an open access article distributed under the terms of the Creative Commons Attribution License (https://creativecommons.org/licenses/by/4.0/). See http://ivyspring.com/terms for full terms and conditions.

Published: 2021.02.20

Corrected article: Int J Biol Sci 2020; 16(11): 1821-1832. doi: 10.7150/ijbs.37552.

In our paper [1], the author has made a mistake by selecting wrong SEM panels during Figure 2 (c, d) and Figure $3(\mathrm{e}, \mathrm{g})$ preparation of 1393@MBG group. Considering the consistency and the accuracy of SEM data, the authors had repeat experiments and carefully re-examined them by SEM for the refabricated 1393(control group) and 1393@MBG scaffolds. The original figures were attached as supplementary figures. The authors apologize for all these errors and state that these corrections do not change the scientific conclusions of the article in any way.

Figure 2, Figure 3 and the legends should be corrected as follows.
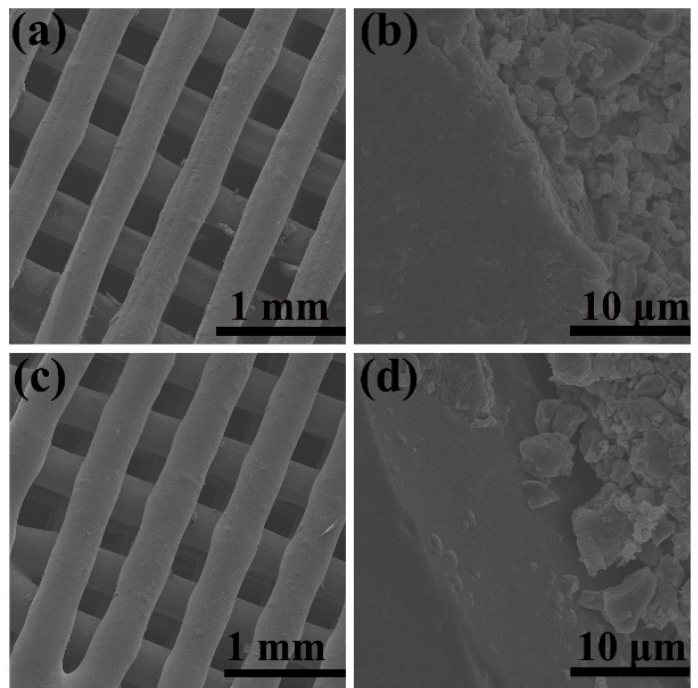

Figure 2. FESEM images of (a, c) as fabricated 1393 and 1393@MBG scaffold; (b, d), the cross section of as fabricated 1393 and 1393@MBG scaffold. 

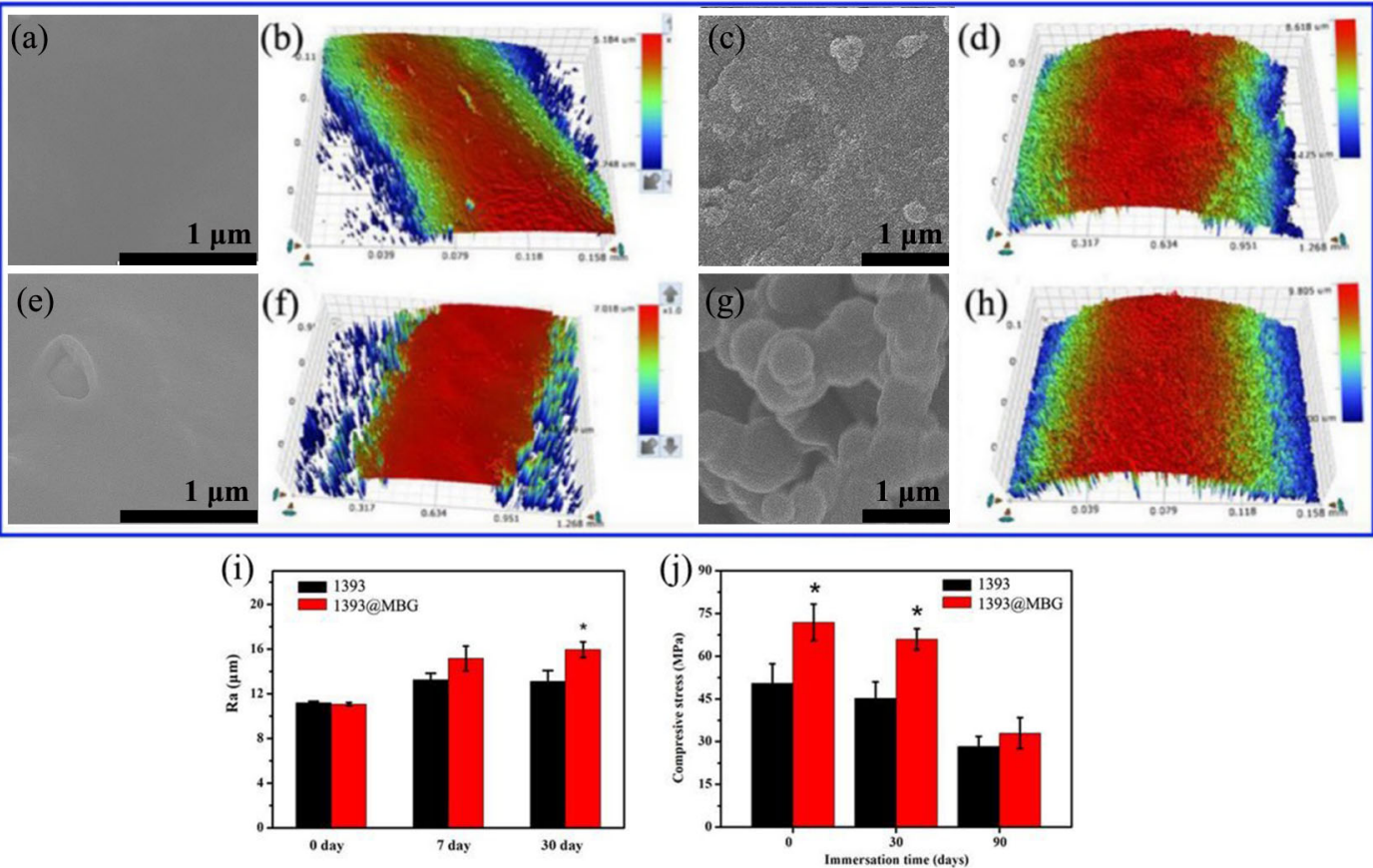

Figure 3. (a, b) FESEM images and the surface profile of as fabricated 1393 scaffold; (c, d) after 30 days immersed scaffold; (e, f) FESEM image and the surface profile of as fabricated 1393@MBG scaffold; (g, h) after 30 days immersed scaffold; (i) The Ra of 1393 and 1393@MBG scaffold surface when immersed from 0 to 30 days; (j) The compressive strength of the 1393 and $1393 @ M B G$ scaffold on day 0,30 and 90 . mean \pm SD, $n=5$. *Significant difference when compared to 1393 ( $p<0.05$ ).

\section{Supplementary Material}

Supplementary figures. http://www.ijbs.com/v17p0913s1.pdf

\section{References}

[1] Wang H, Deng Z, Chen J, Qi X, Pang L, Lin B, Adib YTY, Miao N, Wang D, Zhang Y, Li J, Zeng X. A novel vehicle-like drug delivery 3d printing scaffold and its applications for a rat femoral bone repairing in vitro and in vivo. International journal of biological sciences. 2020;16(11):1821-1832 doi:10.7150/ijbs.37552 